\title{
39
}

\section{Memory effects and microscale}

\author{
Matgorzata Peszyńska \\ Systems Research Institute, Polish Academy of Sciences \\ Newelska 6, 01-447 Warszawa, Poland \\ $E$-mail: mpesz@ibspan.waw.pl
}

\begin{abstract}
In many applications the models describing evolution phenomena with nonlocal effects have the form of PDEs with integral memory terms of Volterra convolution type. In this paper we present an overview of applications and indicate the related analytical and approximation issues. We show that, from computational point of view, in some cases it is advantageous to consider some auxiliary problem defined at microscale which is either imbedded in the definition of the problem or has to be introduced.
\end{abstract}

\section{Keywords}

Integro-partial differential equations, memory terms, microscale, convolution integrals

\section{INTRODUCTION}

In this paper we deal with two intersecting topics: memory effects and microscale. We want to exploit the mutual relation between these two phenomena which frequently are simultaneously present in a model. The purpose of the paper is expository: we present a collection of different results and applications from a new perspective. As the topic is very broad, we will restrict ourselves only to some representative contributions to the field.

We restrict our attention here to the memory effects which arise in evolution equations and have the form of convolution terms

$\mathcal{Q}_{\tau}(u)(t) \stackrel{\text { def }}{=} \tau * \mathcal{D} u=\int_{0}^{t} \tau(t-s) \mathcal{D} u(s) d s$.

Here $u$ denotes the unknown solution to a differential problem (for convenience we omit spatial variables), $\mathcal{D}$ is a differential operator (in applications $\mathcal{D}$ can be the identity operator, the derivative with respect to the time variable, the Laplacian, or some nonlinear elliptic operator). The kernel $\tau: R \mapsto R$ is fixed for a given application and is typically a positive nonincreasing function i.e. the value of the integral depends more on the recent values of $u$ than on the past ones; this property is called fading memory. The function $\tau$ can be bounded or unbounded at the origin; the presence of $\mathcal{Q}_{\tau}(u)$ in an equation affects its solutions in various ways (see section 2).

By microscale we mean any properties of the medium which are observable at a lower scale of observation than the macroscopic equation describing the quantity of interest 
to us. In numerous phenomena there are multiple scales present (micro, meso, macro, giga, ...). Upscaling techniques incorporate the information given on microscale into a law defined at a higher scale; we will denote this by by $m \Rightarrow M$. There exists a variety of upscaling techniques: e.g. the homogenization method, averaging, REV-based methods used in statistical mechanics, methods of asymptotic analysis. For example, the oscillating data of a PDE influence that equation at a microscopic scale while the average (in some sense) of this data enters a (different) PDE at a higher level. In some models we will deal with the coupling between the two scales i.e. $\mathbf{m} \Leftrightarrow \mathbf{M}$.

In some applications, originally of the form $\mathrm{m} \Leftrightarrow \mathbf{M}$, one can define a function $\tau$ and decouple the two scales. The macroscopic problem then is modified by the appearance of a memory term $\mathrm{m} \Rightarrow \tau ; \tau \Rightarrow \mathrm{M}$. At first glance the decoupled system seems attractive from the point of view of analysis and approximation, because when dealing with it we do not have to resolve the complicated coupling on different scales. This approach, however, is only partially advantageous because of the computational issues arising in approximation of memory terms. See below for our model example and later sections 2 and 3 for details. It turns out then that the coupling with microscale can have some advantages over inclusion of memory terms.

Extrapolating this idea, suppose we are given a phenomenon governed by an evolution equation with a memory term $\tau \Leftrightarrow \mathrm{M}$ and that there is no direct relation to any microscale phenomena. For the reasons indicated above we propose to consider construction of some ("artificial") coupling to an auxiliary microscale $\mathbf{m} \Rightarrow \tau$ and then study the complexity of the original problem compared to the one with coupling to microscale, i.e., $\mathbf{m} \Leftrightarrow \mathbf{M}$.

The presentation below starts with a model example. Then in section 2 we discuss various issues related to the analysis and approximation of the memory terms arising in evolution equations. In section 3 we discuss why and how to exploit microscale present in the problem and give a brief review of some models with memory effects and their location in the framework of this paper.

Let us now present a model example. It comes from the study of (single phase, single component) fluid flow through a fissured (fractured, double porosity) medium (see Arbogast (1987), Hornung (1990)) and is derived by the homogenization method. The analysis as well as approximation of the two models as well as of their numerous multiphase and nonlinear extensions have been extensively studied, see references in Hornung (to appear).

$$
\begin{aligned}
\underline{\mathbf{m} \Leftrightarrow \mathbf{M}} & \\
u_{t}-\nabla \cdot(\mathrm{D} \nabla u) & =q_{f m}(x, t), \quad x \in \Omega, t>0, \\
v_{t}^{x}(y, t)-\nabla_{y} \cdot\left(\mathrm{d} \nabla_{y} v^{x}\right) & =0, \quad y \in \Omega_{x}, t>0, \\
\left.v\right|_{\Gamma_{x}} & =u(x, t), t>0, \\
q_{f m}(x, t) & =\frac{1}{\left|\Omega_{x}\right|} \int_{\Omega_{x}} v_{t}^{x}(y, t) d y .
\end{aligned}
$$

The domain $\Omega$ is the macroscopic domain, e.g., a reservoir where the flow of a fluid of density $u$ occurs. At each point $x \in \Omega$ there exists a microscopic domain $\Omega_{x}$ (a porous block) where the flow observable at a lower scale (of density $v^{x}$ ) occurs. All blocks $\Omega_{x}$ are isometric to a certain $\Omega_{0}$. The pair $\left(u,\left\{v^{x}, x \in \Omega\right\}\right)$ gives us the full information about the values of density in the fractures and in the blocks of the fissured medium $\Omega$. The coefficients $\mathbf{D}, \mathbf{d}$ are the mobility coefficients. The equivalent model with explicit memory 
term has the form

$u_{t}-\nabla \cdot \frac{\tau \Leftrightarrow \mathbf{M}}{(\mathrm{D} \nabla u)}=-u_{t} * \tau, x \in \Omega$,

where $\tau$ is obtained from a microscopic block problem

$$
\begin{aligned}
\underline{\mathrm{m} \Rightarrow \tau} & =0, y \in \Omega_{0}, \\
r_{t}-\nabla_{y} \cdot\left(\mathrm{d} \nabla_{y} r\right) & =1, r(y, 0)=0, y \in \Omega_{0}, \\
\left.r\right|_{0} & =-\frac{d}{d t} \frac{1}{\left|\Omega_{0}\right|} \int_{\Omega_{0}} r(y, t) d y . \\
\tau(t) &
\end{aligned}
$$

The values of the kernel $\tau$ in the latter system depend only on the shape of $\Omega_{0}$ and on the coefficient $d$. They can be computed analytically for some particular cases or, in a more general situation, approximated numerically. Once these values have been calculated, the coupling expressed in the first model by the term $q_{f m}$ and boundary values is formally replaced by the memory term $u_{t} * \tau$. Note that by necessity the function $\tau$ is singular at the origin.

At first glance the latter model, as a single parabolic integro-differential equation, seems to be in a more convenient form for mathematical and numerical analysis. Practical evidence however (see Arbogast $(1989,1990)$ and the following papers, references in Hornung (to appear)) suggests that the former (uncoupled) is more appropriate for applications. This is a typical instance of what we want to consider in this paper.

\section{APPROXIMATION}

In this section we want briefly to address the issues that one encounters when dealing with memory terms present in evolution equations: the qualitative and quantitative effect upon the solutions; the design of quadrature rules; the complexity of approximation.

We will consider the following typical cases of the convolution kernels, all of them nonnegative and nonincreasing: (1) trivial; $\tau(t)=0$; (2) bounded; for ex. $\tau(t)=e^{-t}$; (3) unbounded at the origin, $L^{2}(0, T)$ integrable for every $T>0$, for ex. $\tau(t)=t^{-\frac{1}{4}} ;(4)$ unbounded at the origin, non- $L^{2}$ but $L^{1}(0, T)$ integrable, for ex. $\tau(t)=\frac{1}{\sqrt{t}} ;(5)$ unbounded and not integrable near the origin, for ex. $\tau(t)=\frac{1}{t} ;(6)$ extreme; $\tau=\delta\left(\right.$ Dirac) or $\tau=-\delta^{\prime}$, "very" singular at the origin.

If the convolution kernel $\tau$ is $L^{1}(0, T)$, then the convolution operator $\mathcal{Q}_{\tau}$ sending a function $u \mapsto \tau * u$ is linear and continuous on $L^{2}(0, T)$. Additional assumptions on monotonicity of the kernels imply monotonicity of the operator. More precisely, if $\tau$ is an integrable nonconstant function with a continuous negative nondecreasing derivative, then one can prove the following property known as (strong) positivity of the kernel (see MacCamy (1972))

$(u, u * \tau)_{L^{2}(0, T)}>0, u \neq 0, u \in L^{2}(0, T)$. 
This further implies that the operator $\left(I+\mathcal{Q}_{\tau}\right)^{-1}$ is a contraction (see Hornung (1990)). Similar assumptions yield another important property (see Peszynska (to appear))

$\left(u, u_{t} * \tau\right)_{L^{2}(0, T)} \geq-\frac{1}{2}|u(0)|^{2}, u \in L^{2}(0, T)$.

Monotonicity and related properties are used in analysis of the problems with memory terms as well as in proofs of the convergence of the applicable numerical algorithms.

Let us go back to the model example from Introduction. Its well-posedness has been proved with the use of monotonicity techniques mentioned above. The smoothness of the solutions to it is not essentially affected by the presence of the memory terms because, without the coupling term (or memory term, respectively), the equations have purely parabolic character hence "infinite smoothing effects" can be observed. However, the quantative difference in solutions corresponding to the kernels of different degree of singularity is important (see Peszynska (1995)).

The situation changes when the type of the equation in which the convolution term appears is hyperbolic. The results reported in Dafermos (1989) for the viscoelasticity models show that the memory terms contribute to the smoothness of the solutions to these models. This impact becomes stronger with increasing degree of singularity of the convolution kernel, which has the meaning of the growing dissipative part of the equation.

The above phenomenon however, does not make the approximation of memory terms easier as a consequence of increasing degree of singularity of the kernel. The easiest case here is that of bounded kernels and for those most of the work has been done.

More specifically, we consider approximation of the term $\mathcal{Q}_{\tau}(u)(t)$ at $t=t_{N}$ so that $N$ is the number of time steps (of variable or uniform length) that have elapsed. We seek a quadrature rule in the form

$\mathcal{Q}_{\tau}(u)\left(t_{N}\right) \approx \sum_{k=1}^{N} \omega_{N, k}(\mathcal{D} u)_{k}$

In the right rectangular rule, for example, one sets $\omega_{N, k}=\left(t_{k}-t_{k-1}\right) \tau\left(t_{N}-t_{k}\right),(\mathcal{D} u)_{k}=$ $\mathcal{D} u\left(t_{k}\right)$. This rule as well as other typical numerical integration methods (rectangular, trapezoidal) consist in replacing the integrand by its polynomial interpolant. Such an approach is suitable for smooth kernels but fails to guarantee the stability in case of a singular $\tau$. The methods proposed recently in McLean (1993) and Peszynska (to appear), applicable to unbounded kernels, are based on the product integration method. (see Linz (1985)). The idea here is to approximate only the well behaving part of the product and to integrate exactly the remaining part. In our case this would be, respectively, $\mathcal{D} u$ and $\tau$. Additionally some adjustments must be made to make the quadrature rule consistent with the discretization of the original differential equation. One sets then $\omega_{N, k}=$ $\frac{1}{t_{k}-t_{k-1}} \int_{t_{k-1}}^{t_{k}} \int_{0}^{t_{N}} \tau\left(t_{N}-s\right) d s$. For the details (the convergence proof, implementation and applications) see the respective papers.

All of the above mentioned approximation methods have one common characteristic: the weights $\omega_{N, k}$ have to be recomputed at each time step $t=t_{N}$. This implies further that we cannot calculate subtotals for the sum and reuse them at later steps. Rather, we need to store all the information about the "history" of the solution i.e. the values $(\mathcal{D} u)_{1},(\mathcal{D} u)_{2}, \ldots(\mathcal{D} u)_{N}$ in the computer memory. That issue can be critical: note that 
the approximation methods necessary in applications must be combined with some discretization in space. Denote by $N_{h}$ the number of nodes of spatial discretization (for compatibility it can be of order $O(N)$ or $O\left(N^{2}\right)$ in the finite element or finite difference approximation). Then at each time step we need to store vectors of length $N_{h}$. Remembering the whole "history" requires storing $N N_{h}$ numbers. The order of magnitude of $N N_{h}$ may be unacceptably large in a given implementation.

The direct approach to this issue by straightforward "cutting off" the "tail" of the kernel can lead to the loss of accuracy (see Peszynska (to appear)). On the other hand, the use of modern hardware (smart exploitation of different computer memory layers) can help in a particular implementation. The general and safe way to resolve the storage issue was proposed by Thomée and coworkers in Thomee (1992) (see also references in that paper) for bounded kernels and allows for storing only $\sqrt{N} N_{h}$ values of the solution; it is not clear though if that method would work for singular kernels.

In the following section we propose to exploit microscale in order to resolve that complexity problem.

\section{MICROSCALE}

In this section we first briefly show how the use of microscale helps in dealing with the complexity of approximation to the solutions of the model problem. Then we give an overview of applications and study the particular case of the convolution kernels in the form of Prony series. This serves as a motivation for a more general approach.

The solutions to the model problem in the form $\tau \Rightarrow \mathbf{M}$ can be approximated with the use of the algorithm suitable for unbounded kernels (see above) which has, however, the aforementioned drawback of the large storage complexity.

The alternative to the above is given by solving the equivalent problem $\mathbf{m} \Leftrightarrow \mathbf{M}$ where instead of a single memory term one deals with the coupling to microscale. If each node $1 \ldots N_{h}$ of the macro domain $\Omega$ is associated with a copy of microscopic domain $\Omega_{x}$ discretized with $N_{H}$ nodes, then at each time step we need to store $N_{h}$ values of the solution to the $\mathrm{M}$ problem and $N_{H} N_{h}$ values associated with the $\mathrm{m}$ problems. The key point is that $N_{H}$ can be taken relatively small, for example of order 10 . Hence, the storage totals to $\left(N_{H}+1\right) N_{h}$. This is to be contrasted with the number $N N_{h}$ for the model with memory term.

The price we pay for the computer storage savings by using the $\mathbf{m} \Leftrightarrow \mathbf{M}$ approach is a much bigger computational effort: in addition to the macroscopic problem one needs to solve $N_{h}$ microscopic problems at each time step. This overhead can be reduced by solving the $m$ problems (they are independent of each other) in parallel, The use of modern computer architectures is then a major advantage.

Let us now turn back to the general case. Table 1 contains a short overview of applications. The memory terms that arise from microscale are marked with an $\mathbf{m}$ in the first column. For these problems, in case of computer memory storage limitations, one might try to exploit the microscale and compare the efficiency of the two approaches, $\mathrm{m} \Leftrightarrow \mathrm{M}$ and $\tau \Rightarrow \mathrm{M}$. In other problems the memory terms come from constitutive laws and are identified from some empiric data. We shall propose a way to deal with it. As a motivation 


\begin{tabular}{lllll}
\hline $\begin{array}{l}\text { application } \\
\text { how to find } \tau\end{array}$ & $\mathcal{D} u$ & type & refs \\
\hline $\mathbf{m} \quad \begin{array}{l}\text { single phase flow in fissured medium } \\
\text { the use of the heat kernel }\end{array}$ & $u_{t}$ & parabolic & Hornung (1990) \\
\hline $\begin{array}{l}\text { heat cond. in materials with memory } \\
\text { constitutive equations }\end{array}$ & $u_{t}, \Delta u$ & parabolic & Nunziato (1971) \\
\hline $\begin{array}{l}\text { fading memory in viscoelasticity } \\
\text { experimental data fitting }\end{array}$ & $\left(\phi\left(u_{x}\right)\right)_{x}$ & hyperbolic & Dafermos (1989) \\
\hline $\mathbf{m}$ & $\begin{array}{l}\text { homogenization limits of conserv. laws } \\
\text { Young measures }\end{array}$ & $u, u_{x x}$ & $\begin{array}{l}\text { conserv. } \\
\text { laws }\end{array}$ & $\begin{array}{l}\text { Tartar (1990), } \\
\text { Amirat (1989) }\end{array}$ \\
\hline $\begin{array}{l}\text { nonlocal theory of dispersion/diffusion } \\
\text { nonlocal effects in time/space }\end{array}$ & $\Delta u$ & $\begin{array}{l}\text { convection- } \\
\text { diffusion }\end{array}$ & Cushman (1994) \\
\hline $\begin{array}{llll}\text { Fourier and Laplace transform } \\
\text { control theory for phase transitions }\end{array}$ & $\begin{array}{l}\text { general } \\
\text { funct. }\end{array}$ & Stefan pbm & Hoffmann (1991) \\
\hline
\end{tabular}

Table 1 Applications.

let us consider a class of models of consolidation and creep of clay (see Thomas(1989)) where the kernel is sought in the form

$\tau(t)=\sum_{k=1}^{K} \alpha_{k} e^{-\lambda_{k} t}, \alpha_{k}, \lambda_{k}>0$,

(i.e. Prony series). This form is justified by the constitutive construction in which the clay medium behaves like a Hookean spring in series with $K$ Kelvin (i.e. a spring in parallel to a dash-pot) bodies. In general many kernels of the fading memory type are expected to have the form of Prony series, with least-squares fit of the experimental data used to identify the coefficients.

The consequence of such a special form of the kernel is essential. Each term in $\mathcal{Q}_{\tau}(u)(t)=$ $\sum_{k=1}^{K} \alpha_{k}\left(\mathcal{D} u(\bullet) * e^{-\lambda_{k} \bullet}\right)(t)$ can be seen as $\alpha_{k}$ times the solution at time $t$ of an ODE with the stiffness coefficient $\lambda_{k}$ and the right hand side equal to $\mathcal{D} u(t)$. Hence, the value of the memory term can be computed through solutions of these ODEs; their approximation can be calculated by some discretization method appropriate for ODE. We want then to exploit the use of Prony series as some approximate representation of $\tau$. In the framework of this paper that representation can be used to create the coupling with microscale. Microscale here should be understood as $K$ separate ODEs. Note that these ODEs can be solved in parallel and that at each time step we need to store only $K$ values of solutions of ODEs for each point corresponding to the spatial discretization of $\Omega$ i.e. we need to store $K N_{h}$ numbers. This quota should be considerably less than $N N_{h}$ or $\sqrt{N} N_{h}$ storage necessary in case of other methods, and so the microscale approach identified as Prony series looks attractive from the practical point of view. This idea requires careful analysis to be pursued in the forthcoming papers. 
Let us now discuss the potential negative aspects of this approach. These concern mainly the difficulties with finding a proper Prony series and the stiffness of the system of ODEs.

In general, fitting the given experimental data requires solving a large system of nonlinear equations for $\left(\alpha_{k}, \lambda_{k}\right)_{k=1}^{K}$. One way to avoid this as well as to decrease the difficulties with the stiffness of the system of ODEs is by fixing the $\left(\lambda_{k}\right)_{k=1}^{K}$ to be integers from some interval. Suppose the kernel is $L^{2}(0, T)$ integrable. Then, by the change of variable $s=e^{-t}$ the problem of finding $L S Q$ approximation of $\Upsilon(s)=\tau(-\ln s)$ by a polynomial of the form $P(s)=\sum_{k=1} \alpha_{k} s^{\lambda_{k}}$ is well posed. Setting $\lambda_{k}=k$ we need only to find the coefficients $\alpha_{k}$ what can be done by a standard $L S Q$ algorithm. Scaling of the variable $s=e^{-\lambda_{0} t}$ can change the range of sought exponents $\left\{\lambda_{k}\right\}_{k=1}^{K}$. The set of coefficients provides the best LSQ fit to the function $\Upsilon(s)$ and so there is a continuous dependence of the quality of approximation of $\tau(t)$ by $p(t)=P\left(e^{-t}\right)$; the involved constants can be however potentially very large.

Another problem is that the Prony series takes a finite value at the origin equal to $p(0)=\sum_{k=1}^{K} \alpha_{k}$, while in many applications the kernel is unbounded there. However, in the absence of better methods, the approximation of $\tau$ as Prony series doesn't seem to be worse than the application of elementary quadrature schemes to unbounded kernels. The remaining issue is how to properly treat the case $\tau \in L^{1}, \tau \notin L^{2}$.

At the end we want to mention the exact representation of $\tau$ with the use of exponential terms (see Lubich (1988)). This is done with the Laplace transform inversion formula

$\tau(t)=\frac{1}{2 \pi i} \int_{\Gamma} \hat{\tau}(\lambda) e^{\lambda t} d \lambda$

where $\hat{\tau}$ is the Laplace transform of $\tau$ and $\Gamma$ is some appropriately chosen contour in the complex plane. That approach leads however to a quadrature formula, hence, does not resolve the storage complexity problem and cannot be treated as an alternative to the above framework.

\section{ACKNOWLEDGMENT}

The significant part of the research presented in this paper was done while the author was visiting the Center for Applied Mathematics at Purdue University. The author would like to thank Professor Jim Douglas, Jr., the Director of the Center, for his invitation. The research is a result of many discussions at the Center, which the author would like gratefully to acknowledge, in particular with J. Douglas himself, and R.E. Showalter.

\section{REFERENCES}

Amirat, Y., Amdache, K. and Ziani, A. (1989) Homogeneisation d'equations hyperboliques du premier ordre at applications aux ecoulements miscibles en milieux poreux, Ann. Inst. Henri Poincare 6, 397-417

Arbogast T. (1989) Analysis of the Simulation of Single Phase Flow Through a Naturally Fractured Reservoir, SIAM J. Numer. Anal. 26, 12-29 
Arbogast, T., Douglas (Jr.) J. and Hornung U. (1990) Derivation of the Double Porosity Model of Single Phase Flow via Homogenization Theory, SIAM J. Math. Anal. 21, 823-836

Cushman, J.H., Hu, X. and Ginn T.R. (1994) Nonequilibrium Statistical Mechanics of presasymptotic dispersion, Journal of Statistical Physics 75 , 859-878

Dafermos, C., Ericksen, J,L. and Kinderlehrer, D. (1989) Amorphous Polymers and NonNewtonian Fluids, IMA Volumes, Springer-Verlag

Hoffmann, K.H., Kenmochi, N. and Niezgodka, N. (1991) Large Time Solutions of TwoPhase Stefan Problem with Delay, Nonlinear Analysis: TMA

Hornung U. (to appear) Homogenization and Porous Media, Interdisciplinary Applied Mathematics Series, Springer, New York

Hornung, U. and Showalter R.E. (1990) Diffusion Models for Fractured Media, Jour. Math. Anal. Appl. 147, 69-80

Linz P. (1985) Analytical and Numerical Methods for Volterra Equations, SIAM, Philadelphia

Lubich C. (1988) Convolution Quadrature and Discretized Operational Calculus, Parts I E 2, Numer. Math. 52, 129-145 \& 413-425

MacCamy, R.C. and Wong J.S. (1972) Stability Theorems for Some Functional Differential Equations, Trans. Amer. Math. Soc. 164, 1-37

McLean, V., Thomée, V. and Wahlbin L.B. (1993) Discretization with vaiable time steps of an evolution equation with a positive type memory term, Applied Mathematics Report AMR93/18, December 1993, School of Math., The University of New South Wales

Nunziato J.W. (1971) On heat conduction in materials with memory, Quarterly Apppl. Math. 29, 187-204

Peszyńska M. (1995) On a model for nonisothermal flow in fissured media Differential and Integral Equations 8, 1497-1516

Peszyńska M. (to appear), Finite element approximation of diffusion equations with integral convolution terms, Math. Comp.

Tartar L. (1990) Memory effects and homogenization, Arch. Rat. Mech. Anal. 111, 121133

Thomas, H.R. and Bendani K. (1989) Numerical solutions of one-dimensional rheological models of combined consolidation and creep, Eng. Comput. 6, 331-338

Thomée, V. and Wahlbin L.B. (1992) Long time numerical solution of a parabolic equation with memory, Dept. of Math, Chalmers University of Technology, The University of Göteborg, Preprint No 1992-14 